\title{
BMJ Open Impact of healthcare strikes on patient mortality: a protocol for a systematic review and meta-analysis of observational studies
}

\author{
Ryan Essex (D) , ${ }^{1}$ Sharon Marie Weldon, ${ }^{1,2}$ Trevor Thompson (D) , \\ Erika Kalocsanyiova, ${ }^{1}$ Paul McCrone, ${ }^{1}$ Sanjoy Deb ${ }^{3}$
}

To cite: Essex R, Weldon SM, Thompson T, et al. Impact of healthcare strikes on patient mortality: a protocol for a systematic review and metaanalysis of observational studies. BMJ Open 2021;11:e050826. doi:10.1136/ bmjopen-2021-050826

\section{- Prepublication history and} additional supplemental material for this paper are available online. To view these files, please visit the journal online (http://dx.doi.org/10.1136/ bmjopen-2021-050826).

Received 02 March 2021 Accepted 30 November 2021

A) Check for updates

(c) Author(s) (or their employer(s)) 2021. Re-use permitted under CC BY-NC. No commercial re-use. See rights and permissions. Published by BMJ.

${ }^{1}$ Institute of Lifecourse Development, University of Greenwich, London, UK ${ }^{2}$ Barts Health NHS Trust, London, UK

${ }^{3}$ School of Life Sciences, University of Westminster, London, UK

Correspondence to

Dr Ryan Essex;

r.w.essex@gre.ac.uk

\section{ABSTRACT}

Introduction A strike is a collective, temporary and calculated action, which involves a temporary stoppage of work. For healthcare professionals strike action poses a unique dilemma. Perhaps most fundamentally, as strike action is designed to be disruptive it has the potential to impact the delivery of care and place patient well-being in jeopardy. The objective of this study is therefore to evaluate the impact of healthcare strike action on patient mortality outcomes globally using meta-analysis in order to provide a comprehensive evidence base that can advise healthcare professionals, governments and regulatory bodies on the impact that strike action has on patients. Methods and analysis A comprehensive literature search of major electronic databases (EMBASE, MEDLINE, CINAHL, BIOETHICSLINE, EConLit, WEB OF SCIENCE, OPEN GREY and SIGMA REPOSITORY) will be undertaken to identify observational studies of strike action among healthcare professionals where in-hospital/clinic and population/ community mortality is examined, prestrike, during and poststrike. Meta-analysis will be performed to estimate in-hospital/clinic and population/community mortality during periods of strike action. The quality of evidence will be assessed using the National Institute of Health quality assessment tool for observational cohort and crosssectional studies. Risk of bias will be assessed using the Cochrane Risk Of Bias In Non-Randomized Studies - of Interventions tool.

Ethics and dissemination This study does not require ethical approval. Findings will be submitted to an appropriate peer-reviewed journal.

Trial registration number CRD42021238879.

\section{BACKGROUND}

Strike action in healthcare is a remarkably common global phenomenon. A strike (or strike action or labour strike) has been defined as a 'temporary stoppage of work by a group of employees in order to express a grievance or enforce a demand'. ${ }^{1}$ That is, a strike is collective, temporary and calculated, and is largely distinct from other forms of workplace protest and resistance. For healthcare professionals, strike action poses
Strengths and limitations of this study

- This study will outline a systematic review and meta-analysis to assess the effect strike action has on patient and population mortality outcomes.

- Mortality is only one measure of patient well-being/ outcomes-there are other qualitative outcomes which are not included in this meta-analysis.

- It will not be able to fully account for the upstream (or knock-on) effects of strike action. This could be in hospitals where staff did not go on strike or those who did not seek treatment (or sought treatment elsewhere) during the strike.

- This study will not examine the impact of strike action on healthcare delivery (eg, rates of appointments, waiting times, cancellations).

a unique dilemma. Perhaps most fundamentally, as strike action is designed to be disruptive it has the potential to impact the delivery of care and place patient well-being in jeopardy. Beyond this concern, the characteristics of strikes and the patients and healthcare systems they impact vary substantially. Healthcare strikes have been documented on almost every continent, they vary in length, from hours to hundreds of days, ${ }^{2}$ their demands vary ${ }^{3}$ and their impact can be vastly different. ${ }^{4}$ The healthcare systems they impact also vary substantially, from Kenya, to New Zealand to the USA. ${ }^{5}$ Even until recently, strikes have continued to occur throughout the COVID-19 pandemic. ${ }^{6}$

Over the last four decades the justifiability of strike action has been debated in the literature in hundreds of articles, letters, commentaries and debates. Positions on this are often quite polarised, with a number of authors arguing that strike action in healthcare cannot be justified in any circumstance. Counihan, ${ }^{7}$ for example, argues that strike action was akin to 'trying to cure a disease 
by administering poison'. On the other hand, many have argued that strike action is not only permissible but a duty. Brecher, ${ }^{8}$ for example, argues that healthcare professionals are not under any special moral obligation that would prevent them from striking, noting that ' $[u]$ nless we were all either to agree that human life is in all circumstances a completely overriding value ... the striker whose omissions bring about someone's death has no prima facie moral case to answer'. Loewy ${ }^{9}$ builds a similar case, arguing that healthcare professionals are just as essential as those who work in garbage or waste disposal, and that ' $[\mathrm{u}]$ ncollected garbage or unprocessed sewage are every bit as dangerous and have far more sidereaching health effects than do untreated pneumonia or appendicitis or coronary bypass surgeries that are not performed'. Decades later, these discussions remain unsettled and while a number of further ethical considerations can be found throughout the literature, the impact of strike action on patient outcomes, rightly, weighs most heavily. ${ }^{10}$ This largely unsettled dilemma has not only created fertile ground for ongoing ethical discussion, but has left a substantial grey area for healthcare professionals and regulatory bodies who may be considering or facing strike action. For example, during the 2016 UK junior doctors strikes, the General Medical Council (the UK's regulatory body for doctors) issued a stark warning, urging for strike action to be called off, stating that, "we believe that, despite everyone's best efforts, patients will suffer'. ${ }^{11}$

The impact of strike action is measurable and over the last several decades' evidence in relation to such action has expanded. In addition to a broad literature debating the justifiability of strike action, research exists on the impact that strikes have on healthcare delivery, for example, patient presentations and admissions and the attitudes of patients and healthcare professionals toward such action. Given the pressing concerns about patient wellbeing during strike action, an outcome that has received particular attention is patient mortality, with a number of studies measuring patient mortality in-hospital/clinics and in populations/communities during strike action. To a lesser extent, a number of other patient outcomes have been examined in light of strike action, from immunisation rates ${ }^{12}$ to chlamydia rates ${ }^{13}$ for example.

There is a pressing need for clarity in relation to the above literature on patient outcomes. That is, a better understanding of how such action impacts patient mortality (and other outcomes) will have implications for critical normative, practical and regulatory questions related to strike action. Whether such action is justified, how patient well-being can be protected during a strike and whether healthcare professionals should face sanction for participating, are among a number of important questions to which this review will contribute.

The primary objective of this study is therefore to evaluate the impact of healthcare strike action on patient mortality outcomes globally (specifically in-hospital/clinic and population/community mortality captured prestrike, during and in poststrike periods) using meta-analysis in order to provide a comprehensive evidence base that contributes to ethical, practical and regulatory decision making in relation to strike action. The in-hospital/clinic distinction has been made to ensure global inclusivity, for example, some countries do not have hospitals but instead provide healthcare from a clinical setting of sorts such as a rural clinic that serves as a hospital. The population/community distinction has been made in relation to the types of data individual studies may provide.

\section{REVIEW QUESTION}

What is the impact of healthcare strike action on patient mortality outcomes globally?

\section{METHODS}

This protocol conforms to Preferred Reporting Items for Systematic Review and Meta-Analysis Protocols guidelines ${ }^{14}$ for the reporting of systematic review and meta-analysis protocols (see online supplemental file 1). Eligibility criteria were developed using the PECOS (Population, Exposure, Comparator, Outcomes, Study design) framework, ${ }^{15}$ which is summarised in table 1 and described in detail in the later sections.

Table 1 Summary of the PECOS eligibility criteria (detailed descriptions in manuscript text)

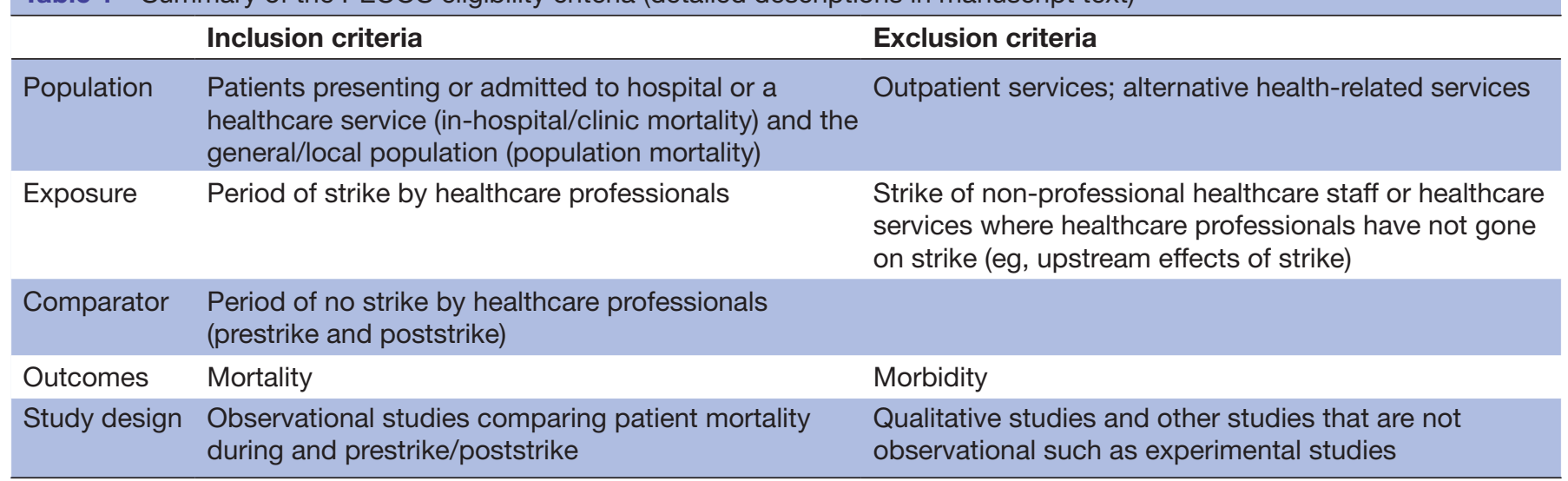




\section{Patient/population}

We will include studies of patients receiving care from hospitals/clinics before, during and after a period of strike (to identify how patients in hospitals/clinics are impacted by strike action). We will include all patients impacted by strike action in-hospital, however, where data exists about patient acuity this will be reported. We will also include studies that examine mortality in the general population/community under study before, during and after a period of strike action (to identify the broader effects of the strike, for example, people who died at home because they didn't seek treatment/ sought treatment elsewhere). For our analysis of in-hospital mortality we have excluded outpatient and alternative health services as in many cases, these services would not be comparable to hospital environments and would have further complicated the results, for example, outpatient and alternative health services are likely to witness far fewer deaths and far less acute patients even outside periods of strike action.

\section{Interventions}

Our intervention event will be strike action by healthcare professionals. Non-professional healthcare strikes will be excluded (eg, porters, drivers, cleaners, administrative staff). When there is a mix of both professional and non-professional healthcare staff striking we will include the study. For these purposes, we will define healthcare professionals as defined in the UK as 'a person associated with either a specialty or a discipline and who is qualified and allowed by regulatory bodies to provide a healthcare service to a patient' ${ }^{16}$ Healthcare professionals will therefore include doctors, nurses, physiotherapists, dieticians and paramedics, among others.

\section{Comparison}

Period of no strike of the same service(s) (prestrike and poststrike); usual care.

\section{Outcomes}

Primary outcome

- In-hospital/clinic mortality.

- In-hospital/clinic mortality during a strike period will be examined against a comparable time period before and/or after the strike action. Mortality rates for each period will be compared.

- Where possible, prestrike and poststrike control periods will be examined to analyse if the strike itself has any effect on patient mortality.

- Population mortality.

- Population mortality during a strike period will be examined against a comparable time period before and/or after the strike action. Mortality rates for each period will be compared.

Note that consideration will be given to the duration of the no strike comparison compared with the strike and whether it is prestrike and poststrike.
Study design

Due to the nature of strikes, this is the best available evidence that can be included in this type of synthesis. Observational studies comparing patient mortality during and prestrike/poststrike. Observational studies can include cohort, longitudinal and cross-sectional designs.

\section{Measures of effect size}

For each study, we will compute the relative risk (RR) of mortality: during versus prestrike/poststrike.

\section{Information sources}

The following electronic databases and time periods will be searched: EMBASE (1980-2021), MEDLINE (1946-2021), CINAHL (1982-2021), BIOETHICSLINE (1972-1999), (This time period has not been limited by the researchers, papers were only indexed in BIOETHICSLINE from 1972 to 1999.) EconLit (1969-2021), WEB OF SCIENCE (1960-2021). In addition, grey literature will be searched through OPEN GREY, and SIGMA REPOSITORY.

Where complete data for a relevant outcome are not available from a report of an eligible study we will contact authors to request data. In addition, we will conduct a manual search of reference lists of eligible studies.

\section{Search strategy}

Search terms have been developed to capture the core concepts, related to the form of intervention we are interested in (eg, strike action, industrial action) and the populations in question (eg, doctors, nurses, healthcare professionals). The final search terms will be: strike OR "industrial action" OR "industrial dispute" OR "collective action” AND doctor OR physician OR clinician OR "medical practitioner" OR nurs* OR "health profession*" OR healthcare OR "health care" OR "pharmac*" OR “dentist" OR "midwi*” OR dieti* OR "occupational therap*" OR "paramed*" OR "physiotherap*" OR "radiograph*” OR "psycholog*” OR "health worker" OR "hospital". There will be no publication dates or language restrictions. If there is a period greater than 3 months from the initial search to journal submission then another search for that time period will be conducted to bring the search up to date. For an expanded search strategy see online supplemental file 2 .

\section{Study selection}

Titles and abstracts of the initial searches will be independently screened by RE and one other member of the review team, who will exclude studies not meeting the eligibility criteria. RE and one other reviewer will then independently screen the full-text of the remaining articles, retaining only eligible studies for inclusion. Disagreements at any stage will be resolved through discussion or with a third member of the review team if necessary. 


\section{CODING AND STATISTICAL ANALYSIS PLAN}

\section{Data extraction}

One reviewer (RE) will perform data extraction, with extracted data checked for accuracy by SMW. Information extracted will include:

- Study characteristics (such as study design, geographical location and year in which the strike took place).

- Overview of the strike characteristics, professionals involved, length of strike and length of control $\operatorname{period}(\mathrm{s})$.

- Mortality outcome results and whether there was adjustment for potential confounders in the analyses.

- Source of data (eg, administrative database, population mortality statistics).

- Any other contextual details about the strike which may be relevant.

\section{Missing data}

Where there is missing data; study authors will be contacted for unreported data and additional details.

\section{Study characteristics}

We will generate a descriptive table summarising the key characteristics of each eligible study including the type of strike, the professionals involved and the patient populations and in-hospital services affected.

\section{Meta-analysis}

Meta-analysis will be used to systematically synthesise the findings of the single, independent studies retrieved from the search and included for analysis. The RR will be calculated for each study. We will pool RRs using a random-effects model and test for heterogeneity (see later). A random-effects method for dichotomous outcomes can combine ORs, risks ratios or risk differences and can be conducted in RevMan where $\log$ transformations can also be conducted. If a metaanalysis is not possible due to too few results or for any other reasons, the authors will assess the suitability of conducting an alternate synthesis.

\section{Analysis of sub-groups}

If possible, we may do subanalysis of the length of strike, professionals involved, services affected and geographical location. We will conduct the subanalysis if there are a minimum of two studies that can be meaningfully pooled and their results are sufficiently similar. If studies are not similar, we will conduct subanalysis if there are four studies. ${ }^{17}$

\section{Heterogeneity}

We will test for variation in effect sizes within a set of studies for the same comparison by computing the $\mathrm{I}^{2}$ statistic, which estimates the proportion of variance in effect sizes due to true heterogeneity. We will also report $\tau$ as a measure of heterogeneity for each comparison, which gives the SD of the effect size estimate.

\section{Sensitivity analysis}

We will assess the robustness of the findings to various types of strikes (length, location, etc) by performing sensitivity analyses including removing studies with a high risk of bias, that is, where overall bias is assessed as being critical using the Risk Of Bias In Non-Randomized Studies - of Interventions (ROBINS-I).

\section{Evidence grading}

The quality of the study evidence for the primary outcome of mortality will be evaluated using the National Institute of Health quality assessment tool ${ }^{18}$ for observational cohort and cross-sectional studies and presented in a Summary of Findings table. Within-study bias will be assessed with the Cochrane ROBINS-I tool, ${ }^{19}$ which rates potential for study bias arising preintervention (confounders, participant selection), during the intervention (classification of intervention) and postintervention (deviations, missing data, outcome measurement, result selection). Two authors will conduct the assessment and a third will look at a random sample assessments.

\section{Patient and public involvement}

This study is a synthesis of secondary data and will not require patient or public involvement.

\section{ETHICS AND DISSEMINATION}

This review does not require ethical approval, as it will use secondary data that is already publicly available. We will disseminate our findings by publishing results in a peerreviewed journal.

Twitter Sharon Marie Weldon @sharonmweldon

Contributors RE conceived the study and developed the study design and protocol. SMW and TT provided substantial contribution to the study design, statistical analysis plan and the writing of the protocol. EK, PM and SD contributed to the design and writing of the protocol. All authors evaluated the study protocol critically for important intellectual content and contributed to its revisions. All the authors gave the final approval to publish the current protocol.

Funding This work is supported by the Institute of Lifecourse Development within the University of Greenwich.

Competing interests None declared.

Patient consent for publication Not applicable.

Provenance and peer review Not commissioned; externally peer reviewed.

Supplemental material This content has been supplied by the author(s). It has not been vetted by BMJ Publishing Group Limited (BMJ) and may not have been peer-reviewed. Any opinions or recommendations discussed are solely those of the author(s) and are not endorsed by BMJ. BMJ disclaims all liability and responsibility arising from any reliance placed on the content. Where the content includes any translated material, BMJ does not warrant the accuracy and reliability of the translations (including but not limited to local regulations, clinical guidelines, terminology, drug names and drug dosages), and is not responsible for any error and/or omissions arising from translation and adaptation or otherwise.

Open access This is an open access article distributed in accordance with the Creative Commons Attribution Non Commercial (CC BY-NC 4.0) license, which permits others to distribute, remix, adapt, build upon this work non-commercially, and license their derivative works on different terms, provided the original work is properly cited, appropriate credit is given, any changes made indicated, and the use is non-commercial. See: http://creativecommons.org/licenses/by-nc/4.0/. 
ORCID iDs

Ryan Essex http://orcid.org/0000-0003-3497-3137

Trevor Thompson http://orcid.org/0000-0001-9880-782X

\section{REFERENCES}

1 Hyman R. Strikes. Springer, 1989.

2 Siegel-Itzkovich J. Israel's doctors take industrial action. BMJ 2000;320:1292.

3 Chatterjee P. India's doctors protest over caste quota plans. Lancet 2006;367:1892-92.

4 Thornton V, Hazell W. Junior doctor strike model of care: reduced access block and predominant fellow of the Australasian College for emergency medicine staffing improve emergency department performance. Emerg Med Australas 2008;20:425-30.

5 Kaguthi GK, Nduba V, Adam MB. The impact of the nurses', doctors and clinical officer strikes on mortality in four health facilities in Kenya. BMC Health Serv Res 2020;20:469.

6 Essex R, Weldon SM. Health care worker strikes and the Covid pandemic. N Engl J Med 2021;384:e93

7 Counihan HE. Industrial action in the health services--the medical perspective. Ir Med J 1982;75:315-7.

8 Brecher R. Striking responsibilities. J Med Ethics 1985;11:66-9.

9 Loewy EH. Of healthcare professionals, ethics, and strikes. Camb Q Healthc Ethics 2000;9:513-20.

10 Essex R, Weldon SM. The justification for strike action in healthcare: a systematic critical interpretive synthesis. Nursing Ethics In Press.
11 Sparrow A. Junior doctors could be struck off over industrial action, warns GMC. The Guardian, 2016. https://www.theguardian.com/ society/2016/sep/05/junior-doctors-could-be-struck-off-overindustrial-action-warns-gmc

12 Njuguna J. Impact of nurses' strike in Kenya on number of fully immunized infants in 18 County referral hospitals. J Health Care Poor Underserved 2018;29:1281-7.

13 Pinto AD, Gournis E, Al-Bargash D, et al. Impact of a labour disruption affecting local public health on the incidence of Chlamydia infections in Toronto. PLoS One 2013;8:e79375.

14 Moher D, Shamseer L, Clarke M, et al. Preferred reporting items for systematic review and meta-analysis protocols (PRISMA-P) 2015 statement. Syst Rev 2015;4:1.

15 The Cochrane CollaborationHiggins JPT, Green S, eds. Cochrane Handbook for systematic reviews of interventions version 5.1.0, 2011. www.handbook.cochrane.org

162011 healthcare professional. (n.d.) segen's medical dictionary. Available: https://medical-dictionary.thefreedictionary.com/ healthcare+professional [Accessed 01 Sep 2021].

17 Ryan R. Cochrane Consumers and Communication Review Group. 'Cochrane Consumers and Communication Group: meta-analysis, 2016. Available: http://cccrg.cochrane.org

18 National Institute for Health. Quality assessment tool for observational cohort and cross-sectional studies, 2014. Available: https://www.nhlbi.nih.gov/health-topics/study-quality-assessmenttools

19 Sterne JA, Hernán MA, Reeves BC, et al. ROBINS-I: a tool for assessing risk of bias in non-randomised studies of interventions. BMJ 2016;355:i4919. 